\title{
Fungsi Zeta Riemann Genap Menggunakan Bilangan Bernoulli
}

\author{
Ikhsan Maulidi ${ }^{*}$, Vina Apriliani' $^{2}$, Muhamad Syazali ${ }^{3}$ \\ ${ }^{1}$ Universitas Syiah Kuala. Jalan Tgk. Nyak Arif, Syiah Kuala, Banda Aceh,23111, Indonesia. \\ 2 Universitas Islam Negeri Ar-Raniry. Jln.Syech Abdurrauf,Syiah Kuala, Banda Aceh, Indonesia \\ 3Universitas Islam Negeri Raden Intan Lampung. Jalan Endro Suratmin, Sukarame, Bandar \\ Lampung 35133, Indonesia. \\ *Corresponding Author. E-mail: ikhsanmaulidi@unsyiah.ac.id
}

\begin{abstract}
Abstrak
Dalam artikel ini kami mengkaji nilai dari Fungsi Zeta Riemann untuk bilangan genap positif menggunakan Bilangan Bernoulli. Di bagian awal kami mengulas sedikit tentang Bilangan Bernoulli dan Fungsi Zeta. Metode yang dilakukan dalam penelitian ini adalah penelitian studi pustaka. Dari hasil analisis yang kami lakukan, diperoleh suatu teorema untuk menghitung nilai Fungi Zeta beserta pembuktian nya.
\end{abstract}

Kata kunci: Fungsi Zeta Riemann, Bilangan Bernoulli, Polinom Bernoulli

\section{Abstract}

In this article, we study about the value of Riemann Zeta Function for even numbers using Bernoulli number. First, we give some basic theory about Bernoulli number and Riemann Zeta function. The method that used in this research was literature study. From our analysis, we have a theorem to evaluate the value of Riemann Zeta function for the even numbers with its proving.

Keywords: Riemann Zeta Function, Bernoulli Number, Bernouli Polynomial.

\section{PENDAHULUAN}

Dalam ilmu matematika, dikenal banyak bilangan khusus yang memiliki banyak terapan atau aplikasi. Salah satu bilangan tersebut adalah bilangan Bernoulli. Bilangan ini ditemukan oleh Jacob Bernoulii (1654-1705 M), seorang matematikawan dan fisikawan berkebangsaan Swiss. Bilangan ini diperoleh dengan diawali dengan diperolehnya suatu bentuk polinom yang disebut Polinom Bernoulli. Bilangan Bernoulli juga memiliki hubungan dengan Bilangan Euler. (Vassilev, 1987) Perumuman dari bilangan ini dapat dilihat pada beberapa artikel (Q.-M. Luo, 2002)

Salah satu manfaat dari ditemukan Polinom dan Bilangan Bernoulli ini adalah untuk menghitung nilai Fungsi Zeta, $\zeta(s)$. Fungsi Zeta ini ditemukan oleh Bernhard
Riemann seorang ilmuwan matematika asal Jerman pada abad ke 19.

Evaluasi nilai dari Fungsi Zeta merupakan hal yang menarik untuk dikaji. Euler dapat memberikan hasil untuk Fungsi Zeta untuk $s=1$. Namun pada saat itu Euler belum dapat menemukan nilai dari fungsi Zeta untuk $s=4$. Fungsi Zeta dapat digunakan untuk menentukan distribusi dari bilangan prima.

Berdasarkan penelitian yang terdahulu fungsi Zeta Riemann telah banyak dilakukan dalam berbagai penelitian (Alabdulmohsin, 2017; Anton, 20114; Atangana \& Noutchie, 2014; Basile, Joung, Lal, \& Li, 2018; Basiuk \& Tarasyuk, 2016; Duong \& Lucchini, 2013; Hasil \& Vesely, 2016; K \& Raina.R.K, 2014; Kim \& Song, 2018; M \& I, 2010; Pan \& Chen, 2011; R. Sita Rama Chandra RAO \& Sarma, 2015; Riguidel, 2018; Wang, Liu, \& 
Hou, 2018; Williams, 2015; Wu \& Bercu, 2017; Xin, 2016; Xu, 2016; Yildiz, Tunc, \& Kavurmaci, 2014). Namun belum terdapat penelitian yang mengkaji nilai dari Fungsi Zeta Riemann untuk bilangan genap positif menggunakan Bilangan Bernoulli. Maka, dalam artikel ini diberikan salah satu cara menentukan nilai Fungsi Zeta untuk nilai $s$ bilangan genap yaitu dengan menggunakan Bilangan Bernoulli. Dengan pendekatan Bernoulli ini, diyakini dapat mempercepat proses komputasi dibandingkan dengan menggunakan cara biasa atau secara rekursif.

\section{METODE}

Penelitian ini merupakan penelitian studi pustaka dan dilakukan pada November 2018 sampai Desember 2018. Tempat penelitian ini adalah di Perpustakaan Universitas Syiah Kuala dan Laboratorium Komputasi Numerik.

Dalam penelitian ini diberikan salah satu cara lain dalam pembuktian Teorema yang disajikan dengan menggunakan kajian konsep Polinom Bernoulli yang ada dalam penlitian terdahulu (Kelly, W. G, Peterson, 2001) Bukti lain dari Teorema ini dapat dilihat pada penelitian oleh Silva (Silva, 2006)

\section{HASIL DAN PEMBAHASAN}

Sebelum membahas Teorema inti dalam artikel ini, berikut kami sajikan beberapa Definisi dan Lema yang relevan dan berguna dalam memahami konsep yang ada.

\section{Definisi 3.1 (Polinom Bernulli)}

Suatu poinom Bernoulli, $B_{k}(t)$, didefinsikan sebagai berikut :

$$
\frac{x e^{t x}}{e^{x}-1}=\sum_{k=0}^{\infty} \frac{B_{k}(t)}{k !} x^{k} .
$$

Dengan kata lain $\frac{x e^{t x}}{e^{x}-1}$ merupakan fungsi pembangkit eksponensial untuk barisan $B_{k}(t)$.
Dengan mengekspansi kedua ruas diperoleh beberapa Polinom Bernoulli berikut:

$B_{0}(t)=1$.

$B_{1}(t)=t-\frac{1}{2}$.

$B_{2}(t)=t^{2}-t+\frac{1}{6}$.

$B_{3}(t)=t^{3}-\frac{3}{2} t^{2}+\frac{1}{2} t$.

(Kelly, W. G, Peterson, 2001)

\section{Definisi 3.2 (Bilangan Bernoulli)}

Bilangan Bernoulli ke $k, B_{k}$, didefinisikan dengan mengevaluasi poinom Bernoulli ke $k$ untuk $t=0$, atau

$$
B_{k}=B_{k}(0) \text {. }
$$

Beberapabilangan Bernoulli,

$B_{0}=B_{0}(0)=1$,

$B_{1}=B_{1}(0)=0-\frac{1}{2}=-\frac{1}{2}$,

$B_{2}=B_{2}(0)=0^{2}-0+\frac{1}{6}=\frac{1}{6}$,

$B_{3}=B_{3}(0)=0$.

\section{Definisi 3.3 (Operator Beda)}

Operator beda, $\Delta$, didefinisikansebagai

$$
\Delta y(t)=y(t+1)-y(t) \text {. }
$$

\section{Lema $\quad 3.1 \quad$ (Sifat-sifat Polinom Bernoulli)}

Berikut ini adalah beberapa sifat dari Polinom Bernoulli:

3.1.a $B_{k}^{\prime}(t)=k B_{k-1}(t), \quad k \geq 1$.

3.1.b $\Delta_{t} B_{k}(t)=k t^{k-1}, \quad k \geq 0$.

Bukti 3.1.a:

Dengan menurunkan kedua ruas persamaan (1) terhadap $t$ diperoleh

$$
\begin{gathered}
\frac{x^{2} e^{t x}}{e^{x}-1}=\sum_{k=0}^{\infty} \frac{B_{k}^{\prime}(t)}{k !} x^{k}, \\
x \sum_{k=0}^{\infty} \frac{B_{k}(t)}{k !} x^{k}=\sum_{k=0}^{\infty} \frac{B_{k}^{\prime}(t)}{k !} x^{k}, \\
\sum_{k=0}^{\infty} \frac{B_{k}(t)}{k !} x^{k+1}=\sum_{k=0}^{\infty} \frac{B_{k}^{\prime}(t)}{k !} x^{k},
\end{gathered}
$$




$$
\sum_{k=1}^{\infty} \frac{B_{k-1}(t)}{(k-1) !} x^{k}=\sum_{k=1}^{\infty} \frac{B_{k}^{\prime}(t)}{k !} x^{k},
$$

Sehingga $\frac{B_{k-1}(t)}{(k-1) !}=\frac{B_{k}^{\prime}(t)}{k !}, B_{k}^{\prime}(t)=$ $k B_{k-1}(t), k \geq 1$. Terbukti.

Bukti3.1.b

Denganmengambilbedadarikeduaruas pada persamaan (1),

$$
\begin{gathered}
\Delta_{t} \sum_{k=0}^{\infty} \frac{B_{k}(t)}{k !} x^{k}=\Delta_{t} \frac{x e^{t x}}{e^{x}-1} . \\
\sum_{k=0}^{\infty} \Delta_{t} \frac{B_{k}(t)}{k !} x^{k}=\frac{x}{e^{x}-1}\left(e^{(t+1) x}-e^{t x}\right) \\
=\frac{x}{e^{x}-1} e^{t x}\left(e^{x}-1\right)=x e^{t x} \\
\sum_{k=0}^{\infty} \Delta_{t} \frac{B_{k}(t)}{k !} x^{k}=\sum_{k=0}^{\infty} \frac{t^{k} x^{k+1}}{k !}
\end{gathered}
$$

Yang setaradengan

$$
\sum_{k=1}^{\infty} \Delta_{t} \frac{B_{k}(t)}{k !} x^{k}=\sum_{k=1}^{\infty} \frac{t^{k-1} x^{k}}{(k-1) !}
$$

Sehingga $\Delta_{t} \frac{B_{k}(t)}{k !}=\frac{t^{k-1}}{(k-1) !} \Delta_{t} B_{k}(t)=$ $k t^{k-1}$, untuk $k \geq 1$. Jelas pula bahwa $\Delta_{t} B_{0}(t)=0$, jadi $\Delta_{t} B_{k}(t)=$ $k t^{k-1}$, untuk $k \geq 0$.

\section{Definisi 3.4 (Fungsi Zeta Riemann)}

Fungsi Zeta Riemann adalah fungsi yang didefinisikan sebagai berikut:

$$
\zeta(s)=\sum_{n=1}^{\infty} \frac{1}{n^{s}} .
$$

Evaluasi nilai Fungsi Zeta Riemann untuk $s$ bilangan genap daat diperoleh dengan melakukan transformasi Polinom Bernoulli ke dalam bentuk Deret Fourier. Deret ini dikembangkan oleh Joseph Fourier dimana menggunakan deret tak hingga dari fungsi sinus dan kosinus.

\section{Lema 3.2 (Deret Fourier pada Interval} $\left[-\frac{T}{2}, \frac{T}{2}\right]$ )

Deret Fourier darifungsi $f(x)$ dengan periode $T$ pada interval $\left[\frac{-T}{2}, \frac{T}{2}\right]$ didefinisikan sebagai deret

$$
\begin{aligned}
F(x)=\frac{a_{0}}{2}+ & \sum_{n=1}^{\infty} a_{n} \cos \left(\frac{2 n \pi x}{T}\right) \\
& +\sum_{n=1}^{\infty} b_{n} \sin \left(\frac{2 n \pi x}{T}\right),
\end{aligned}
$$

dimana

$$
a_{n}=\frac{1}{T} \int_{\frac{-T}{2}}^{\frac{T}{2}} f(x) \cos \left(\frac{2 n \pi x}{T}\right) d x
$$

dan

$$
b_{n}=\frac{1}{T} \int_{\frac{-T}{2}}^{\frac{T}{2}} f(x) \sin \left(\frac{2 n \pi x}{T}\right) d x .
$$

(Young, 1992)

\section{Lema 3.3 (Beberapa Sifat Deret Fourier )}

Diberikanekspansi Fourier $F(x)=$ $\sum_{n=1}^{\infty} a_{n} \cos \left(\frac{2 n \pi x}{T}\right)+\sum_{n=1}^{\infty} b_{n} \sin \left(\frac{2 n \pi x}{T}\right)$ untuk fungsi $f(x)$. Maka berlaku sifatsifat berikut:

a. Jika $f(x)$ adalah fungsi ganjil $(f(-x)=-f(x))$ maka $a_{n}=0$;

b. Jika $f(x)$ adalah fungsi genap $(f(-x)=f(x))$ maka $b_{n}=0$;

c. $\int_{-\infty}^{\infty}|f(x)|^{2} d x=\frac{1}{T} \int_{-\infty}^{\infty}|F(x)|^{2} d x$ (Teorema Parseval).

\section{Pembahasan Fungsi Zeta Riemann Genap}

Teorema 1 (Fungsi Zeta Riemann Genap) Untuksuatubilanganpositif $k$ berlaku

$$
\zeta(2 k)=\sum_{n=1}^{\infty} \frac{1}{n^{2 k}}=\frac{(-1)^{k-1} B_{2 k}(2 \pi)^{2 k}}{2(2 k) !} .
$$

\section{Bukti :}

Diawalidenganmenyatakanfungsi $B_{1}(x)=$ $x-\frac{1}{2}$ pada $\left[-\frac{1}{2}, \frac{1}{2}\right]$ sebagai Deret Fourier. Untuk mempermudah misalkan $B_{1,1}(x)=$ $x$, jelas bahwa fungsi $B_{1,1}$ adalah fungsi ganjil, sehingga $a_{n}=0$ untuk $n \geq 1$.

Sementarauntuk $T=1, a_{0}=$ $\frac{1}{1} \int_{-\frac{1}{2}}^{\frac{1}{2}} f(x) \cos (2 n \pi x) \mathrm{dx}=\frac{1}{1} \int_{-\frac{1}{2}}^{\frac{1}{2}} x d x=0$.

Karena $f(x)=x$ adalah fungsi ganjil maka $a_{n}=0, n \geq 1$. Selanjutnya 


$$
b_{n}=\int_{\frac{-1}{2}}^{\frac{1}{2}} x \sin (2 n \pi x) x=\frac{(-1)^{n+1}}{n \pi} .
$$

Maka fungsi $f(x)=x$ untuk $x \in$ $\left[-\frac{1}{2}, \frac{1}{2}\right]$ dapat dinyatakan dengan

$$
f(x)=x=\sum_{n=1}^{\infty} \frac{(-1)^{n+1}}{n \pi} \sin (2 n \pi x) .
$$

Dengan melakukan translasi dari $\left[-\frac{1}{2}, \frac{1}{2}\right]$ ke $[0,1]$,

$$
x-\frac{1}{2}=\sum_{n=1}^{\infty} \frac{(-1)^{n+1}}{n \pi} \sin \left(2 n \pi\left(x-\frac{1}{2}\right)\right) \text {, }
$$

diperoleh

$$
x=\sum_{n=1}^{\infty} \frac{(-1)^{n+1}}{n \pi} \sin \left(2 n \pi\left(x-\frac{1}{2}\right)\right)+\frac{1}{2} .
$$

Sehingga polinom Bernoulli $B_{1}(x)$ dapat dinyatakan dalam deret Fourier berikut

$$
B_{1}(x)=\sum_{n=1}^{\infty} \frac{(-1)^{n+1}}{n \pi} \sin \left(2 n \pi\left(x-\frac{1}{2}\right)\right) \text {. }
$$

Dari Teorema3.1.a dan Teorema Dasar Kalkulus

$$
\begin{aligned}
& 2 \int_{0}^{x} B_{1}(t) d t=B_{2}(x)-B_{2}(0)= \\
& -2(2)\left(\sum_{n=1}^{\infty} \frac{(-1)^{n+1}}{(2 n \pi)^{2}} \cos \left(2 n \pi\left(x-\frac{1}{2}\right)\right)\right. \\
& \left.-\sum_{n=1}^{\infty} \frac{(-1)^{n+1}}{(2 n \pi)^{2}} \cos (-n \pi)\right) \\
& B_{2}(x)=-2 \sum_{n=1}^{\infty} \frac{(-1)^{n+1}}{(2 n \pi)^{2}} \cos (2 n \pi(x \\
& \left.\left.-\frac{1}{2}\right)\right) .
\end{aligned}
$$

Dengan mengintegralkan $B_{2}(x)$ dua kali diperoleh

$$
\begin{aligned}
& B_{4}(x)=(4 !) 2 \sum_{n=1}^{\infty} \frac{(-1)^{n+1}}{(2 n \pi)^{4}} \cos (2 n \pi(x \\
&\left.\left.-\frac{1}{2}\right)\right) .
\end{aligned}
$$

Jika proses ini kita ulangi terus maka akan diperoleh polaberikut

$$
\begin{aligned}
& B_{2 k}(x) \\
& =2(2 k !)(-1)^{k} \sum_{n=1}^{\infty} \frac{(-1)^{n+1}}{(2 n \pi)^{2 k}} \cos (2 n \pi(x \\
& \left.\left.-\frac{1}{2}\right)\right)
\end{aligned}
$$

Untuk $k \geq 1$.

Subtitusikan $x=0$ ke persamaan (..) diperoleh

$$
\begin{gathered}
B_{2 k}(0) \\
=2(2 k !)(-1)^{k} \sum_{n=1}^{\infty} \frac{(-1)^{n+1}}{(2 n \pi)^{2 k}} \cos (-n \pi) \\
B_{2 k}=2(2 k !)(-1)^{k+1} \sum_{n=1}^{\infty} \frac{1}{(2 n \pi)^{2 k}} \\
(-1)^{k+1} \frac{B_{2 k}(2 \pi)^{2 k}}{2(2 k !)}=\sum_{n=1}^{\infty} \frac{1}{n^{2 k}}=\zeta(2 k) .
\end{gathered}
$$

Terbukti.

\section{ContohAplikasi Teorema 1}

Dengan mengetahui nilai $B_{2}=\frac{1}{6}$, kita dapat menghitung nilai $\sum_{n=1}^{\infty} \frac{1}{n^{2}}$ seperti berikut

$$
\begin{aligned}
\sum_{n=1}^{\infty} \frac{1}{n^{2}}=\zeta(2) & =(-1)^{1+1} \frac{B_{2}(2 \pi)^{2}}{2(2 !)}=\frac{\frac{1}{6} 4 \pi^{2}}{4} \\
& =\frac{\pi^{2}}{6} .
\end{aligned}
$$

Berikut kami sajikan beberapa nilai Fungsi Zeta genap

\begin{tabular}{ccc}
\hline $\mathrm{k}$ & $B_{2 k}$ & $\zeta(2 k)=(-1)^{k+1} \frac{B_{2 k}(2 \pi)^{2 k}}{2(2 k !)}$ \\
\hline 1 & $\frac{1}{6}$ & $\frac{\pi^{2}}{6}$ \\
2 & $-\frac{1}{30}$ & $\frac{\pi^{2}}{360}$ \\
3 & $\frac{1}{42}$ & $\frac{\pi^{2}}{15120}$ \\
4 & $-\frac{1}{30}$ & $\frac{\pi^{2}}{604800}$ \\
5 & $\frac{5}{66}$ & $\frac{\pi^{2}}{23950080}$ \\
\hline
\end{tabular}

SIMPULAN DAN SARAN 
Berdasarkan hasil pembahasan, Nilai Fungsi Zeta Riemann untuk bilangan genap dapat ditentukan dengan menggunakan Bilangan Bernoulli. Hasil ini disajikan dalam Teorema 1 beserta bukti nya.

Berdasarkan hasil penelitian, selain untuk Fungsi Zeta Riemann genap perlu dikaji juga bagaimana cara menentukan nilai Fungsi Zeta Riemann ganjil

\section{DAFTAR PUSTAKA}

Alabdulmohsin, I. M. (2017). Fractional parts and their relations to the values of the Riemann Zeta Function. Arabian Journal of Mathematics, 7(1), 1-8.

Anton, D. (20114). Fourier expansion along geodesics on Riemann surfaces. Open Mathematics, 12(4), 559-573.

Atangana, A., \& Noutchie, O. (2014). A Modified Groundwater Flow Model Using the Spce Time RiemannLiouville Fractional Derivatives Spproximation. Abstract and Applied Analysis, 2014.

Basile, T., Joung, E., Lal, S., \& Li, W. (2018). Character Integral representation of Zeta Function in AdSd+1 Part 1 Derivation of The General Formula. Journal Of High Energy Physics, 2018(10), 1-35.

Basiuk, Y. ., \& Tarasyuk, S. . (2016). Fourier Coefficients Assoxiated With The Riemann Zeta Function. Karpats'ki Matematicni Publikaci, 8(1), 16-20.

Duong, H. D., \& Lucchini, A. (2013). A Finiteness condition on the coefficients of the probabilistic zeta function. International Journal of Group Theory, 2(1), 167-174.

Hasil, P., \& Vesely, M. (2016). Oscillation and non-oscillation criterion for Riemann- Weber type half-linier differential equations. Electronic Journal of Qualitative Theory of
Differential Equationd, 2016(12), 122.

K, P. R., \& Raina.R.K. (2014). On a Certain Extension of The Hurwitz Lerch Zeta Function. Annals of the West University of Timisoara, 52(2), 157170.

Kelly, W. G, Peterson, A. . (2001). Difference Equation: An Introduction with Applications. Florida: Harcourt.

Kim, D., \& Song, K. (2018). The Inverses of Tails of The Riemann Zeta Function. Journal of Inequalities and Applications, 2018(1), 1-13.

M, H., \& I, H. I. (2010). A Derivation Of Spin Based on a Stability Analysis of The Riemann-Zeta Function. Progress in Physics, 2010(2), 55-55.

Pan, T., \& Chen, H. (2011). Two Dimention Riemann Initial-Boundary Value Problem of Scalar Conservation Laws with Curved Boundary. Boundary Value Problems, 20(11), 1-16.

Q.-M. Luo, B.-N. G. F. Q. (2002). Generalizations of Bernoulli's numbers and polynomials.

R. Sita Rama Chandra RAO, A., \& Sarma, S. R. (2015). Some Identities Involving The Riemann Zeta Function. In Procceding of Indian National Science Academy.

Riguidel, M. (2018). Morphogenesis of The Zeta Function in the Critical Strip by Computational Approach. Mathematics, 6(12), 285.

Silva, J. B. (2006). Bernoulli Numbers and their Applications.

Vassilev, M. V. (1987). Relations between Bernoulli numbers and Euler numbers.

Wang, Y., Liu, Y., \& Hou, C. (2018). New Concept of Fractional Hahns Omega Derivative of Riemann-Liouville type and Caputo type and Application. Advances in Difference Equations, 2018(1), 1-21.

Williams, F. L. (2015). Zeta Function Expression of Spin Partition Functions on Thermal AdS3. 


\section{Desimal, 2 (1), 2019 - 48}

Ikhsan Maulidi, Vina Aprilian, Muhamad Syazali

Mathematics, 3(3), 653-665.

Wu, S., \& Bercu, G. (2017). Fast Convergence of Generalized DeTemple Sequences and The Relation to the Riemann Zeta Function. Journal of Inequalities and Applications, 2017(1), 1-10.

Xin, L. (2016). Some Identities Related to Riemann Zeta Function. Journal of Inequalities and Applications, 2016(1), 1-6.

$\mathrm{Xu}, \quad$ H. (2016). Some Computational Formulas Related The Riemann ZetaFunction Tails. Journal of Inequalities and Applications, 2016(1), 1-7.
Yildiz, C., Tunc, M., \& Kavurmaci, H. (2014). Inequalities for Co-ordinated m-Convex Functions via RiemannLiouville Fractional Integrals. International Journal of Analysis and Application, 5(1), 45-55.

Young, R. M. (1992). Excursions in calculus: an interplay of the continuous and the discrete Washington, D.C. Washington, D.C: Mathematical Association of America, c1992. 